\title{
Fonoaudiologia e Núcleos de Apoio à Saúde da Família: conceitos e referências
}

\author{
Speech-Language Pathology and Centers for Supporting the \\ Family Health: concepts and references
}

\author{
Daniela Regina Molini-Avejonas¹, Vera Lúcia Ferreira Mendes², Cibelle Albuquerque de la Higuera Amato ${ }^{3}$
}

\begin{abstract}
RESUMO
Visando dar subsídios à análise do trabalho do fonoaudiólogo na Atenção Básica, mais especificamente nos Núcleos de Apoio à Saúde da Família (NASF), este artigo realizou revisão da literatura sobre o tema abordado usando ferramentas de busca do Medline, SciELO e o LILACS, por intermédio da pesquisa avançada simultânea de base de dados da Biblioteca Virtual em Saúde (BVS), da Bireme. Procurou-se resgatar um pouco da história da Fonoaudiologia no SUS e dar ênfase à importância da Estratégia de Saúde da Família relacionada ao serviço de Fonoaudiologia nos NASFs. A pesquisa realizada aponta que a atuação da Fonoaudiologia dentro do sistema de saúde é bastante extensa, perpassando diferentes níveis de assistência, o que requer o desenvolvimento de distintas tecnologias de trabalho em saúde. As ações desenvolvidas pelos NASFs exigem, necessariamente, a atuação de equipes interdisciplinares, implicadas em processos de trabalho transdisciplinar e na criação/invenção de dispositivos terapêuticos. A análise das experiências de atuação da Fonoaudiologia na Atenção Básica, na Saúde da Família e, mais recentemente, nos NASFs, merece ser acompanhada e avaliada de perto para que se possa avançar na produção e sistematização de práticas de cuidado à saúde que respondam às necessidades da população e que sejam capazes de enfrentar os graves efeitos dos distúrbios de linguagem, voz, motricidade oral e audição. Desta forma conclui-se que se faz necessário o monitoramento e sistematização dos limites e potencialidades deste modelo, visando seu aprimoramento e apropriação do mesmo, como estratégia de atenção integral, de inserção da Fonoaudiologia no SUS.
\end{abstract}

Descritores: Saúde pública; Atenção primária à saúde; Saúde da família; Sistema único de saúde; Promoção da saúde

\section{INTRODUÇÃO}

Os Núcleos de Apoio à Saúde da Família (NASF), recentemente criados pela portaria ministerial $154 / 2008$, têm por objetivo ampliar a abrangência e o escopo das ações da atenção básica, bem como sua resolutividade, apoiando a inserção da estratégia de Saúde da Família na rede de serviços e o processo

Trabalho realizado em colaboração no Curso de Fonoaudiologia da Faculdade de Medicina da Universidade de São Paulo - FMUSP - São Paulo (SP), Brasil, e no Curso de Fonoaudiologia da Faculdade de Ciências Humanas e da Saúde da Pontifícia Universidade Católica de São Paulo - PUC-SP - São Paulo (SP), Brasil.

(1) Doutora, Professora do Departamento de Fisioterapia, Fonoaudiologia e Terapia Ocupacional da Faculdade de Medicina da Universidade de São Paulo - USP - São Paulo (SP), Brasil.

(2) Doutora, Professora Assistente da Faculdade de Ciências Humanas e da Saúde da Pontifícia Universidade Católica de São Paulo - PUC-SP - São Paulo (SP), Brasil.

(3) Doutora, Fonoaudióloga do Departamento de Fisioterapia, Fonoaudiologia e Terapia Ocupacional da Faculdade de Medicina da Universidade de São Paulo - USP - São Paulo (SP), Brasil.

Endereço para correspondência: Daniela Regina Molini-Avejonas. R. Cipotânea, 51, Cidade Universitária, São Paulo (SP), Brasil, CEP: 05360-160. E-mail: danielamolini@usp.br

Recebido em: 8/5/2010; Aceito em: 25/7/2010 de territorialização e regionalização a partir da atenção básica.

A análise das experiências de atuação da Fonoaudiologia na Saúde da Família e, mais recentemente nos NASFs, merece ser acompanhada e avaliada de perto, uma vez que estamos diante de uma das propostas mais promissoras de ampliação do acesso da população à Atenção Integral à Saúde.

Sabemos que a produção dos processos de cuidado à saúde vem passando por uma série de inflexões, exigindo dos profissionais a incorporação de saberes sobre o trabalho em saúde e o desenvolvimento de novas competências e habilidades, entre as quais destacam-se a capacidade de trabalho em equipe e de criar e conduzir projetos terapêuticos a partir dos conceitos de clínica ampliada e do apoio matricial. Também exige a apropriação de um novo campo conceitual expresso nas diretrizes das políticas públicas do SUS.

Visando dar subsídios à análise do trabalho do Fonoaudiólogo na Atenção Básica, mais especificamente nos NASFs, este artigo realizou revisão da literatura sobre o tema abordado através de ferramentas de busca do Medline, SciELO e o LILACS por intermédio da pesquisa avançada simultânea de base de dados da Biblioteca Virtual em Saúde (BVS) da Bireme, além de livros e jornais de referência. Procurou-se resgatar um pouco da história da Fonoaudiologia no SUS e dar ênfase 
à importância da Estratégia de Saúde da Família relacionada ao serviço de Fonoaudiologia nos NASFs. Foram utilizados os seguintes descritores: Fonoaudiologia e saúde da família. Utilizou-se como critério de inclusão para a seleção das referências a adequação ao tema pesquisado, excluindo-se aquelas que não se encaixavam com exatidão ao tema em questão.

\section{REVISÃO DE LITERATURA}

A Constituição Brasileira de 1988 garantiu a saúde como um direito social dos cidadãos brasileiros. O Sistema Único de Saúde (SUS) apresentado pela Lei Orgânica da Saúde, Lei 8080 de 19 de setembro de 1990, reconhece-o como uma política pública promotora de cidadania pela sua grande capacidade de inclusão e amplitude de suas ações. A participação organizada da população, ou seja, dos usuários, nos mais distintos setores de composição do SUS foi institucionalizada pela Lei 8142 de 28 de dezembro de $1990^{(1)}$.

A proposta do SUS com relação ao cuidado a saúde é ordenada em níveis de atenção (básica, média complexidade e de alta complexidade), tendo todos a mesma relevância sob a compreensão de que a atenção à saúde precisa ser integral. Dentro desta perspectiva, a organização do sistema de saúde não mais é pensada na ótica da organização piramidal, sendo substituída pela proposição de construir redes de cuidado à saúde, conforme ilustrado na Figura $1^{(2)}$.

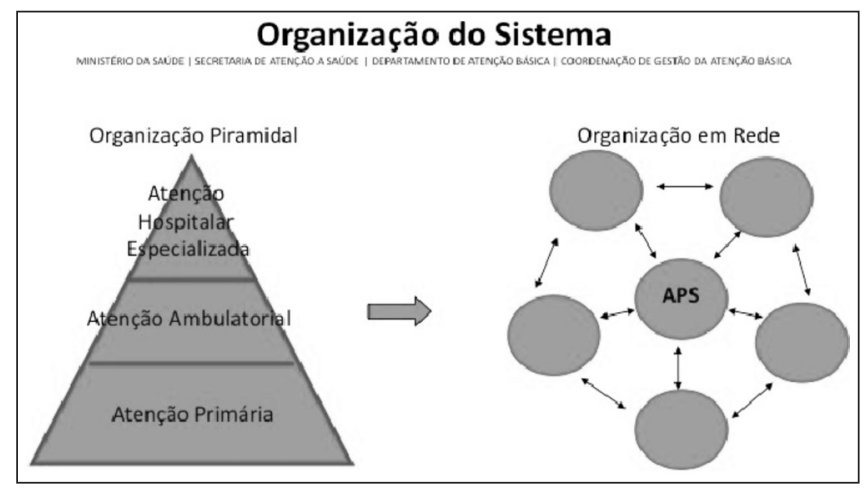

Fonte: Ministério da Saúde, 2006(2)

Figura 1. Organização do Sistema de Saúde.

A Atenção Básica envolve um conjunto de ações promotoras de saúde tanto no contexto individual como no coletivo e atua na promoção e na proteção da saúde, na prevenção de agravos, no diagnóstico, tratamento, reabilitação e na manutenção da saúde. Nessa dimensão, a incorporação do conceito ampliado de saúde, que a associa às condições de vida, norteia a formulação e a implementação de estratégias que viabilizem um serviço de saúde universal, integral, eficaz, eficiente, com equidade e participação popular fez-se necessária ${ }^{(3)}$.

O Ministério da Saúde, visando melhorar os indicadores de saúde da população, principalmente nas regiões do país que apresentam baixa oferta de serviços de saúde e que manifestavam maiores índices de risco e vulnerabilidade social; implementou, a partir de 1990, o Programa de Agentes Comunitários (PAC). No nordeste brasileiro, tais experiências demonstram-se exitosas, proporcionando a sustentação dos referenciais técnico-operacionais do Programa e a expansão da Estratégia de Saúde da Família (ESF) em diversas regiões do país ${ }^{(4)}$.

Em 1994, a Saúde da Família (ESF) chega a grandes centros urbanos (São Paulo, Rio de Janeiro e Porto Alegre) e o impacto desta na melhoria das condições de saúde de indivíduos e grupos sociais não apenas se confirmaram, como ganharam grande repercussão no país, configurando-se assim, em uma das prioridades do Ministério da Saúde como política de qualificação da atenção básica ${ }^{(5)}$.

A Saúde da Família, segundo a Política Nacional de Atenção Básica (PNAB/2006), tem como principal propósito "consolidar e qualificar a estratégia da Saúde Família como modelo de Atenção Básica e centro ordenador das redes de atenção à saúde no Sistema Único de Saúde (SUS)”. Apresenta os seguintes fundamentos: a) efetivar a integralidade de forma interdisciplinar e com coordenação do cuidado na rede de serviços; $b$ ) desenvolvimento de práticas em saúde a partir do diagnóstico do território local, tendo como foco a família e a comunidade e, ainda, que preconizem o vínculo, a co-responsabilização e a atenção continuada; c) criação e/ou aprimoramento de sistemas de avaliação e monitoramento dos indicadores de saúde; d) ampliar o acesso universal e contínuo a serviços de saúde; e) desenvolver ações intersetoriais e a integração co instituições e organizações sociais; f) estimular a participação popular e controle social, como espaço de construção de cidadania. Propõe novas bases para a atenção à saúde de caráter substitutivo ao modelo tradicional a partir da noção de Clínica Ampliada, conforme podemos verificar na Figura $2^{(6)}$ :

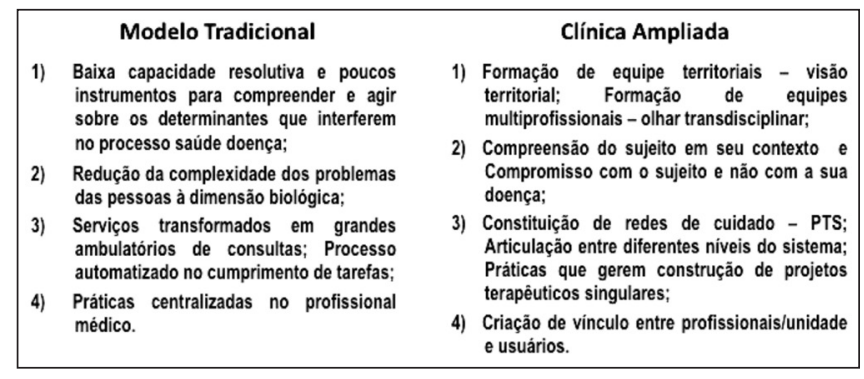

Fonte: Mendes, 2009(6)

Figura 2. Caracterização do modelo tradicional e clínica ampliada.

A Atenção Primária como orientadora do Sistema de Saúde é a tendência registrada nos principais países do mundo, porque alcançam melhores resultados em saúde, trazem maior satisfação aos usuários, menores custos e maior equidade ${ }^{(7)}$.

Estudos internacionais têm evidenciado que a atenção primária tem impacto positivo sobre vários indicadores de saúde; reduz gastos totais; melhora o acesso a serviços de saúde, a qualidade global da atenção à saúde e as ações preventivas; facilita a detecção precoce de problemas reduzindo internações hospitalares; garante cuidados mais apropriados e reduz o uso de cuidados desnecessários de atenção especializada à saúde. Estudos no Brasil apontam na mesma direção: a Saúde da Família melhora o acesso e tem alguns efeitos positivos na situação de saúde ${ }^{(3)}$. A implantação tem ocorrido de forma gradativa, marcada pelo aumento da cobertura das equipes de Saúde da Família em todos os estados da federação( ${ }^{(8)}$. 
Países orientados para a Atenção Primária apresentam menor número de crianças com baixo peso ao nascer, menor mortalidade infantil, especialmente pós-neonatal, menor perda de anos de vida devido a suicídio e maior expectativa de vida ${ }^{(7,9)}$, o que justifica a Saúde da Família como estratégia preconizada pelo Ministério da Saúde como estruturante para a Atenção Primária à Saúde no Brasil ${ }^{(10)}$.

Vários autores investigaram a percepção dos profissionais de saúde, gestores e usuários sobre o sistema público de saúde. Em um dos estudos realizados, todos os gestores tiveram expectativas favoráveis quanto ao desempenho da Atenção Básica e da Saúde da Família. Para os entrevistados, a ESF pode aumentar a eficácia dos serviços públicos e contribuir para a estruturação decorrente da mudança do modelo assistencial para o cuidado integral da saúde favorecendo desta forma a melhoria dos indicadores epidemiológicos. As principais dificuldades para a maioria dos gestores no que diz respeito à organização da Atenção Básica foram entre outros a falta de qualificação dos profissionais envolvidos e a escassez de recursos financeiros ${ }^{(11)}$.

Em outro estudo realizado em Minas Gerais, os gestores acreditam que apesar dos fundamentos da ESF estarem definidos e bastantes claros, principalmente no que se refere às ações de prevenção e promoção à saúde, grande parte das vezes, a atuação dos profissionais envolvidos restringe-se às ações curativas, o que demonstra a importância das políticas de educação permanente e de mudanças nos processos de formação do profissional de saúde. Aparecem, também, relatos de que a própria população, capturada pela lógica hospitalocêntrica, apresenta certa dificuldade na apreensão deste novo referencial $^{(12)}$.

Um dos objetivos da Estratégia de Saúde da Família é construir um novo paradigma para os processos de cuidado à saúde, que considere a família como parte integrante do processo, no qual os profissionais visualizem e integrem-se na diversidade e complexidade do mundo familiar. Dentro desta perspectiva a Saúde da Família é uma estratégia promissora para favorecer encontros entre profissionais e usuários, buscando assim reconhecer a evidência das possibilidades de risco vivida pelo grupo familiar ${ }^{(13)}$.

A ESF apóia-se na organização territorial para planejar sua atuação. Os serviços da atenção primária são relacionados ao conceito que a saúde está construída de uma maneira que não possa ser separada da região em que o usuário habita ${ }^{(14)}$.

De acordo com estudo realizado para avaliar a implantação da ESF observou-se que a implantação ocorreu de maneira adequada e ampliou o acesso da população ao sistema de saúde. Entretanto observou-se falhas no acesso da população ao sistema (relação entre o número de equipes disponíveis e o número de famílias para serem atendidas) ${ }^{(15)}$.

Foi aplicado um questionário em funcionários e usuários do sistema público de saúde e ambas as categorias definiram como quatro principais pontos para atenção primária em saúde: o acesso aos serviços, a coordenação e continuidade da equipe de atenção primária com os outros níveis assistenciais, relação entre profissionais e usuários e a qualidade técnico-científica das equipes de atenção primária ${ }^{(16)}$. Para atingir aos objetivos à que se propõe, em todo o mundo a Atenção Primária em Saúde é organizada obedecendo a alguns princípios fundamentais que organizam o sistema da saúde. O primeiro deles, e a porta de entrada com objetivo de prevenir intervenções desnecessárias; o segundo é a longitudinalidade (continuidade do cuidado, com o desenvolvimento de uma relação interpessoal entre profissionais e pacientes ao longo do tempo); o terceiro, a integralidade (com a disponibilização de diferentes recursos para abordagem de forma adequada e completa às necessidades de saúde do paciente e da comunidade) e por último, a coordenação do cuidado, com o acompanhamento do paciente mesmo que seja tratado por outro nível de atenção.

No estudo em que a unidade de análise foi a Equipe de Saúde da Família do município de Natal (RN) os resultados apontaram para um predomínio da equipe do tipo integração. $\mathrm{O}$ trabalho em equipe surgiu como elemento essencial para o desenvolvimento da Estratégia de Saúde da Família ${ }^{(17)}$.

$\mathrm{O}$ atendimento é prestado na unidade básica de saúde ou em domicílio, pelos profissionais da Equipe de Saúde da Família (EqSF) composta por médicos, enfermeiros, auxiliares de enfermagem e agentes comunitários de saúde. Além disso, há a atuação das equipes de saúde bucal e saúde mental, sendo que esta última foi recentemente incorporada à equipe do NASF, juntamente com outros profissionais de saúde, entre os quais figura o Fonoaudiólogo ${ }^{(1,18)}$.

A Portaria Ministerial 154/2008 considerou a necessidade de agregar outras profissões da saúde que comporiam o NASF. São constituídos por equipes compostas por profissionais escolhidos entre 13 diferentes ocupações, compartilhando com as Equipes de Saúde da Família as práticas em saúde nos territórios sob sua responsabilidade, aumentando seu alcance e eficiência ${ }^{(4)}$.

Duas modalidades de núcleos foram instituídas. O NASF do tipo um é composto por, no mínimo, cinco profissionais de ocupações não coincidentes, vinculados a pelo menos oito equipes de Saúde da Família. O NASF dois é composto por, no mínimo três profissionais de áreas não coincidentes, vinculados a três equipes de Saúde da Família. O gestor local é quem define o elenco dos profissionais do NASF, de acordo com a necessidade da população. Em ambos, o fonoaudiólogo pode integrar a equipe ${ }^{(4)}$.

Em junho de 2009 somavam 648 NASF em funcionamento no país, representando um marco importante na ampliação das possibilidades de alcançar melhores resultados em saúde, com o enfoque na promoção da saúde e no cuidado à população. Com a inclusão dos novos profissionais de saúde, vinculados às Equipes de Saúde da Família, aumenta-se a possibilidade de responder aos novos e antigos desafios da morbidade dos brasileiros. O NASF traz a possibilidade de ampliar a oferta das práticas integrativas e complementares e a oferta da melhor tecnologia disponível para grande parte das doenças crônicas, bem como a revisão dos tratamentos baseados somente na medicalização das doenças ${ }^{(19)}$.

A criação dos Núcleos de Apoio à Saúde da Família se constitui como um passo importante para a consolidação da Estratégia de Saúde da Família e especialmente para o desenvolvimento e aprimoramento de um novo modelo de exercício do trabalho em equipe multiprofissional. Nele, as diretrizes da integralidade, qualidade, eqüidade e participação social devem 
ser concretizadas em ações coletivas centradas no desenvolvimento humano e na promoção da saúde, capazes de produzir saúde para além do marco individualista e assistencialista. $\mathrm{O}$ projeto é ambicioso e pode parecer utópico quando avaliamos que o perfil formativo da maior parte dos profissionais de saúde ainda está muito distante desse horizonte e que muitos obstáculos serão encontrados no percurso ${ }^{(20)}$.

A proposta do NASF vai de encontro a uma lacuna do Sistema Nacional de Saúde. "A falta de filtro na atenção primária não só aumenta consideravelmente o número de consultas desnecessárias, como também sobrecarrega a atenção secundária e aumenta os riscos para os pacientes. Nos centros urbanos brasileiros é comum as pessoas (com ou sem acesso ao sistema privado) preferirem buscar diretamente ao especialista." O autor complementa que a utilização de tecnologias econômicas eficazes como grupos de orientação associadas a profissionais bem treinados e infra-estrutura básica podem promover uma alta resolução de casos com baixos custos ${ }^{(11)}$.

Os Núcleos visam fortalecer oito diretrizes na atenção à saúde: a interdisciplinaridade, a intersetorialidade, o território, a integralidade, o controle social, a educação permanente em saúde, a promoção da saúde e a humanização, atuando em oito áreas estratégicas: atividade física/práticas corporais; práticas integrativas e complementares; reabilitação; alimentação e nutrição; saúde mental; serviço social; saúde da criança, do adolescente e do jovem; saúde da mulher e assistência farmacêutica $^{(20)}$.

Os NASFs vieram para contribuir com as equipes de ESF, tendo como preceito básico um trabalho interdisciplinar e de matriciamento, respeitando e atuando de acordo com as peculiaridades regionais para se ter uma promoção da saúde mais eficiente, com profissões e áreas que até então não eram contempladas no âmbito das equipes de $\mathrm{ESF}^{(21)}$.

$\mathrm{O}$ trabalho em equipe, ainda pouco discutido em pesquisas na saúde coletiva, representa um dos principais pilares da Saúde da Família. A perspectiva da integralidade das ações favorece uma ação inter/transdisciplinar. A consolidação do projeto deve ser norteado por um trabalho assistencial comum onde haja interação entre os agentes e a comunidade envolvida ${ }^{(17)}$.

Os NASFs não foram criados para servir de porta de entrada para o usuário dentro do sistema de saúde, mas para atuar de forma integrada à rede a partir das demandas identificadas no trabalho conjunto com as equipes de Saúde da Família, garantindo uma interface entre ambos. Integrantes das equipes e dos núcleos de apoio compartilham a atenção às famílias ${ }^{(5)}$.

A operacionalização do NASF adota, entre algumas concepções, o conceito de matriciamento, tendo por objetivo garantir que as equipes estabeleçam relações ou responsabilizem-se pelas ações desencadeadas, garantindo a integralidade da atenção em todo o sistema de saúde ${ }^{(6)}$.

Entende-se por matriciamento, arranjos organizacionais que buscam diminuir a fragmentação dos processos de trabalho decorrente da especialização crescente das áreas do cuidado, proporcionando a construção de momentos relacionais onde se estabelece a troca de saberes entre os profissionais de diferentes áreas, conforme descrito na Figura $3^{(6)}$.

O que se pretende, quando a equipe ou profissional de apoio matricial (do NASF) se encontra com a equipe de referência,

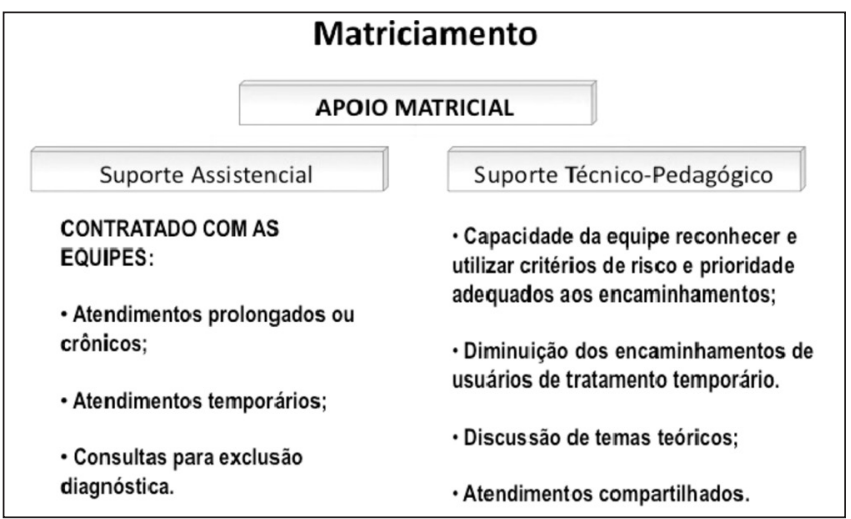

Fonte: Mendes, 2009(6)

Figura 3. Descrição de matriciamento.

é que o apoio matricial auxilie a equipe de referência na formulação/reformulação e execução de um projeto terapêutico singular para um sujeito individual ou coletivo, que necessita de uma intervenção em saúde, à qual a equipe de referência teve dificuldade. Este processo contribui para organização de uma linha de cuidado contínua, rompendo com a fragmentação do cuidado, que prejudica a integralidade da atenção ${ }^{(19)}$.

O projeto Terapêutico ${ }^{(6)}$ é um movimento de co-produção e co-gestão do cuidado de um Sujeito Singular. Pressupõem a discussão coletiva de caso clínico; realização de reunião de toda equipe para definição de propostas de ações; considerar que todas as opiniões são importantes para compreender o caso. São organizados em quatro momentos: 1) Diagnóstico, buscando uma avaliação que possibilite uma conclusão a respeito do risco e da vulnerabilidade do usuário; 2) Definição de Metas, visando construir propostas de curto médio e longo prazo; 3) Divisão de Responsabilidades, onde compartilha-se ou defini-se as tarefas de cada um com clareza, aumentando as possibilidades de identificação dos problemas e diminuindo as possibilidades das práticas meramente prescritivas; 4) Reavaliação, momento em que se discutirá a evolução e se farão as devidas correção de rumo.

Em pesquisa desenvolvida sobre matriciamento, os autores observaram que o mesmo aumentou a resolutividade e a co-responsabilidade dos serviços, promoveu a construção de protocolos de registro e de formas de organização do fluxo da demanda a partir da definição de critérios e criação de práticas de cuidado com o envolvimento dos diferentes serviços da rede. Algumas ações fonoaudiológicas foram fortalecidas: participação nas reuniões de equipe; discussão de casos e construção de projetos terapêuticos conjuntos; valorização da referência qualificada; capacitação em serviço; consulta em conjunto; e ações intersetoriais ${ }^{(22)}$. Estes dados corroboram achados de outro estudo ${ }^{(23)}$.

Segundo os mesmos autores, ainda faz-se necessário o monitoramento e sistematização das ações e dos limites e potencialidades deste modelo, visando seu aprimoramento e apropriação do mesmo como estratégia de atenção integral, de inserção da Fonoaudiologia no SUS e de ampliação do acesso da população a este serviço ${ }^{(22)}$.

O Ministério da Saúde recomenda uma revisão da prática dos encaminhamentos. A maior parte do tempo dos profissio- 
nais do NASF não deverá ser dedicada ao atendimento direto da população, mas a outras atividades junto às equipes. Em lugar do encaminhamento direto, no modelo de referência e contra-referência, o que é recomendado é o compartilhamento de projetos terapêuticos, a atenção domiciliar e a educação permanente $^{(5)}$.

O cuidado domiciliar envolve compreender que a família, a vida doméstica e a comunitária não são isoladas, mas inseridas na dinâmica política e econômicas da sociedade como um todo. Assim, os conflitos sociais de classe, raça, sexo, também devem ser abordados pelos trabalhadores da saúde na sua prática. O cuidado no espaço domiciliar inclui fortalecer relações familiares, respeitar vínculos afetivos e redes de solidariedade social específicas de cada local ${ }^{(24)}$.

Com a revisão da prática do encaminhamento com base nos processos de referência e contra-referência, amplia-se o trabalho para um processo de acompanhamento longitudinal do usuário de responsabilidade da equipe de Atenção Primária/ Saúde da Família, atuando no fortalecimento de seus atributos e no papel de coordenação do cuidado no SUS ${ }^{(25)}$.

Dentro desta perspectiva, a visão clínica tradicional, na qual o atendimento fonoaudiológico direto ao paciente é a atividade prioritária é ampliada. Os núcleos formados por profissionais de diversas áreas deverão dar prioridade a atividades conjuntas de projetos terapêuticos. Reuniões periódicas e visitas aos pacientes indicados pelas ESF fazem parte da rotina dos núcleos, que atenderão às mais diversas solicitações ${ }^{(25)}$.

A atuação da Fonoaudiologia dentro do Sistema de saúde é bastante extensa, perpassando diferentes níveis de assistência, o que requer o desenvolvimento de distintas tecnologias de trabalho em saúde que sejam capazes de cuidar dos processos de adoecimento de pessoas e de grupos sociais de risco ou em situação de vulnerabilidade social e de desenvolver ações de promoção, prevenção e educação em saúde, diretamente relacionadas à melhoria dos indicadores de qualidade de vida e de saúde da população(26).

É dentro do NASF que o fonoaudiólogo generalista encontrará seu maior campo de atuação. Deve organizar suas práticas em bases territoriais definidas a partir do conhecimento das necessidades de saúde da população e de perfis epidemiológicos específicos, sendo responsável pelos processos de cuidado de $90 \%$ dos agravos à saúde ${ }^{(5)}$.

As ações aqui desenvolvidas exigem, necessariamente, a atuação de equipes interdisciplinares, implicadas em processos de trabalho transdisciplinar e na criação/invenção de dispositivos terapêuticos, o que o coloca diante de uma outra ética na produção dos saberes, das práticas e das relações no campo da saúde, capazes de fomentar trocas solidárias em redes transdisciplinares; implicando gestores, profissionais e usuários em processos humanizados de produção dos serviços ${ }^{(26)}$.

Um exemplo do que vem sendo dito até agora é o trabalho realizado em uma universidade de Santa Catarina. Na atenção básica, atua-se utilizando a ESF na área circunvizinha à universidade, área esta bastante heterogênea tanto do ponto de vista de necessidades de saúde quanto de risco epidemiológico, o que justificou a criação e atuação do NASF pela necessidade de apoio multiprofissional e interdisciplinar para as equipes da ESF. Esta iniciativa foi pioneira no município e procura dar suporte para as equipes da ESF, ampliando a resolubilidade e avançando na construção da integralidade das ações de saúde. Dentre as atividades desenvolvidas pela Fonoaudiologia junto à equipe contemplam-se visitas domiciliares, discussão de caso, educação em saúde, atividades em grupo na unidade de saúde e nas instituições pertencentes à área de abrangência das equipes, participação no grupo de saúde mental e atuação em sala de espera ${ }^{(23)}$.

É importante destacar que o objetivo do NASF não é servir como referência para a Atenção Básica, mas sim estar integrado a esta, evitando a necessidade de posterior consulta em nível secundário e possibilitando, não apenas aos profissionais que dele fazem parte, mas também, aos diferentes alunos da universidade, a observação dos diferentes saberes e o modo de fazer das diferentes profissões na área da saúde. Essa experiência de atuação tem auxiliado a construir as possibilidades de trabalho da Fonoaudiologia na atenção básica e vivenciar os embates e enfrentamentos necessários a construção de saberes coletivos e interdisciplinares ${ }^{(23)}$.

Existem inúmeros desafios para o profissional do NASF. As realidades que os profissionais vão encontrar diferem bastante da prática clínica diária, seja ela privada ou em ambulatório público. Existe a necessidade de apropriação de novas técnicas de trabalho junto às equipes de Saúde da Família e à comunidade, como a clínica ampliada (com a consulta conjunta), o projeto terapêutico singular e o apoio matricial. As equipes do NASF precisam se apropriar de novas técnicas, porque mais de $80 \%$ do seu tempo de trabalho deve ser dedicado nesse atendimento junto às equipes e não apenas com os pacientes ${ }^{(10)}$.

A demanda por reflexão e diálogo em torno dessa perspectiva nova ocorre tanto na esfera interna de cada profissão quanto no campo da saúde como um todo. Por um lado, é preciso que os profissionais reflitam conjuntamente sobre as finalidades de sua prática, reconstruindo o sentido e o significado do seu agir, tornando-os coerentes com a proposta apresentada, em especial no que tange à atenção básica. Por outro, torna-se indispensável a ponderação, junto a gestores e organizações comprometidas com a participação social, sobre a relação entre a composição das equipes e as necessidades de saúde das comunidades ${ }^{(27)}$.

Os fonoaudiólogos necessitam ter disponibilidade para atuação a partir da necessidade da população local e não do interesse próprio ou formação/especialização. Conhecimentos na área de saúde pública e saúde coletiva são essenciais para dar subsídios teórico-práticos. Ainda há muita ênfase na formação de especialistas em detrimento de generalistas, mas ambos são importantes ${ }^{(28)}$.

Outro desafio a ser vencido é a criação de especialização ou residência multiprofissional em Saúde da Família e a capacitação dos profissionais que já entraram no NASF para que ofereçam uma atuação adequada às necessidades da população ${ }^{(10)}$.

Durante a implantação dos NASF várias são as etapas a serem cumpridas. Um estudo sugere, por exemplo, nos primeiros meses a realização do mapeamento das áreas de atuação, obtendo informações sócio-demográficas e epidemiológicas. Após mapeamento, a realização de grupos de orientação com a população e também com os demais profissionais da saúde 
sobre a atuação de cada especialidade. Depois do trabalho de divulgação do NASF, iniciam-se os trabalhos específicos de prevenção e promoção da saúde. A Fonoaudiologia realiza palestras, oficinas e grupos de orientação sobre desenvolvimento da linguagem, audição e das funções estomatognáticas, e em casos nos quais é identificada a necessidade de reabilitação, encaminha para clínicas credenciadas do município ${ }^{(29)}$.

A integração dos profissionais da equipe e a articulação com os setores da administração pública e demais parceiros é de fundamental importância para o sucesso do trabalho de promoção de saúde e prevenção de agravos. As autoras do estudo afirmam que as ações fonoaudiológicas estão sendo repensadas e aprimoradas com vistas a acompanhar a evolução do modelo de atenção à Saúde, sendo direcionadas cada vez mais para a promoção da saúde e diminuindo o enfoque dado à atuação clínica ${ }^{(29)}$.

Em um estudo de acompanhamento, objetivando apresentar o programa de cuidado domiciliar, os resultados indicaram limitações na identificação das pessoas que poderiam ser incluídas no programa das Unidades Básicas de Saúde; dificuldades na implantação de rotinas e avaliação do programa; dilemas éticos em relação ao cuidado que o familiar idoso recebe dos seus cuidadores; necessidade do atendimento interdisciplinar e obstáculos à viabilização das internações domiciliares. A parceria terapêutica entre profissionais e usuários foi um aspecto positivo. A população em cuidado domiciliar totalizou 110 pessoas, em onze meses de programa, predominando idosos e mulheres. As necessidades de cuidado mais freqüentes foram com relação a idosos com limitações físicas e sequelados por acidente vascular cerebral. As parcerias de cuidado entre residentes se deram mais frequentemente com cuidadores mulheres, do lar, filhas ou esposas ${ }^{(24)}$.

Estudo desenvolvido por fonoaudiólogos com sete equipes de saúde da família demonstrou que o incentivo e assistência à prática do aleitamento materno (AM), como uma das principais ações que devem ser desenvolvidas pelas Equipes de Saúde da Família (ESF) no seguimento ambulatorial de recém-nascidos, especialmente os de baixo peso ao nascer, contribuem para redução da morbi-mortalidade dessa população e favorece a melhor qualidade de vida desses lactentes ${ }^{(30)}$.

A composição de cada NASF considera estudos epidemiológicos para saber da necessidade de cada região. Partindo deste esquema geral, cada equipe lida com as pecularidades de sua região. A começar pela composição da equipe. Tudo depende das características de cada território. Eé este território, enquanto campo dinâmico das relações, que direciona o tipo de atuação do $\mathrm{NASF}^{(25)}$.

Com o objetivo de verificar qual a necessidade específica fonoaudiológica da população da região Sudeste de São Paulo e de traçar estratégias para atender as necessidades dessa população, foi desenvolvido um levantamento da demanda populacional que necessitava da intervenção do fonoaudiólogo do NASF. Tal levantamento se deu por meio de reuniões mensais com 26 Equipes de Saúde da Família (ESF) e em reuniões quinzenais com Fórum Saúde e Educação, conhecido no território como "Pólo das Escolas", onde profissionais da saúde e educação discutem casos de crianças das escolas do território. Observou-se que a busca dos usuários para tal espe- cialidade se dá principalmente devido a distúrbio fonológico em crianças de faixa etária entre 4 e 6 anos, que frequentam as EMEIs (Escola Municipal de ensino Infantil) do território. Essa demanda de crianças vem encaminhada direta ou indiretamente pelas escolas do território. Após o levantamento da demanda foi desenvolvido o projeto de atendimento específico fonoaudiológico em grupo para melhor atender a necessidade dessa população e outra estratégia ainda em desenvolvimento foi o trabalho voltado às EMEIs envolvidas, através de palestras de conscientização e atualização. Concluímos assim, que o papel fundamental do fonoaudiólogo do NASF nesse território é desenvolver projetos de busca ativa nessas escolas para então desenvolver atividades de prevenção e recuperação da alteração acima citada. É importante referir que essas escolas do território estão necessitando de ações curativas, contudo se não ações de prevenção não forem iniciadas, somente os sintomas do problema serão trabalhados e não a causa destes. O desafio do fonoaudiólogo do NASF junto ao PSF, neste território é: trabalhar com a ESF, as escolas e a população a cultura da promoção e da prevenção da saúde com o objetivo de minimizar tais demandas levantadas atingindo a população ainda em seu estado saudável ${ }^{(31)}$.

Pelas características da população atendida, o acolhimento, que é um facilitador do acesso ao serviço de saúde e oferecimento da assistência adequada, coloca-se como ferramenta ainda mais essencial, assim como a flexibilidade e inteligência coletiva dos trabalhadores de saúde em compor propostas alternativas que possibilitem a aproximação da comunidade e o desenvolvimento do cuidado ${ }^{(25)}$.

Entre as várias responsabilidades atribuídas aos integrantes dos NASFs pela Portaria 154, estão a integração às diversas políticas sociais, atenção humanizada, identificação, em conjunto com as ESF e a comunidade, das atividades, das ações, do público prioritário e das práticas a serem adotadas em cada uma das áreas cobertas; atuação de forma integrada e planejada nas atividades desenvolvidas pelas ESFs e de Internação Domiciliar, quando estas existirem, acompanhando e atendendo a casos, de acordo com os critérios previamente estabelecidos, além da elaboração de projetos terapêuticos individuais, por meio de discussões periódicas que permitam a apropriação coletiva pelas ESF e os NASFs do acompanhamento dos usuários, realizando ações multiprofissionais e transdisciplinares, desenvolvendo a responsabilidade compartilhada ${ }^{(32)}$.

Isso significa pensar em nova forma, na qual os profissionais atuem compartilhando e potencializando o conhecimento e as ações com aumento das trocas e organização do cuidado com descentralização do poder, ou seja, em cooperação, articulação, conexão e rede, que inclui a possibilidade também do confronto intra ou interinstitucional e da divergência de visões no cuidado como estruturante do trabalho e da equipe como um todo ${ }^{(25)}$.

Nem sempre essa equipe funcionará de maneira articulada como demonstram dados de pesquisa realizada em uma UBS da Zona Norte. O PSF foi inserido nesta UBS há dez anos e atualmente conta com cinco equipes de saúde da família que acompanham cerca de 4500 pessoas. Os estagiários de Fonoaudiologia prestam assistência através de grupos terapêuticos, oficinas de linguagem, grupos programáticos e atendimentos 
em visitas domiciliares. Através de observações, do levantamento do Sistema de Informação de Atenção Básica (SIAB) do período de janeiro a maio de 2008 pôde-se verificar uma maciça participação da comunidade nos programas desenvolvidos pela UBS - grupos programáticos, campanhas, oficinas, entre outros. Porém observou-se que o número de adolescentes e pré-adolescentes que freqüentam a Unidade é baixo, assim como a freqüência dos pacientes aos grupos terapêuticos fonoaudiológicos. Um aspecto importante a ser relatado foi o grau de interesse dos agentes comunitários de saúde (ACSs) no acompanhamento e discussão dos atendimentos domiciliares realizados pela Fonoaudiologia. Embora em muitos casos, este trabalho gere um processo de capacitação e de espaço de formação do ACS, também é bastante significativo o número de ACSs que não se implicam no processo de trabalho dos atendimentos domiciliares. Conclui-se que novas redes de cuidados devem ser criadas com a função de atingir adolescentes e pré-adolescentes. Bem como, de ampliar as discussões e resgatar a importância do acompanhamento do ACSs junto aos atendimentos domiciliares e também das possibilidades de atuação do fonoaudiólogo junto a estes $\operatorname{casos}^{(1)}$.

As ações previstas pela Portaria são detalhadas para cada uma das oito áreas estratégicas citadas anteriormente. Dentre elas há destaque para necessidade das ações em saúde mental com a recomendação de que tais atividades devem integrar-se à rede de serviços evitando respostas simplificadas em procedimentos psiquiátricos e medicalizantes. Podemos salientar também as ações de reabilitação que abrangem as pessoas com deficiência com a priorização das ações de inclusão social. A entrada dessas duas grandes áreas, saúde mental e reabilitação, nas atribuições dos NASF supre uma lacuna que inicialmente não havia sido prevista no campo da atenção básica e deveria ser priorizada, pois refere-se à grupos populacionais ainda insuficientemente acolhidos pelas ações das políticas públicas ${ }^{(20)}$.

A Portaria também define o papel do profissional de reabilitação como aquele que promova "ações que propiciem a redução de incapacidades e deficiências com vistas à melhoria da qualidade de vida dos indivíduos, favorecendo sua reinserção social, combatendo a discriminação e ampliando o acesso ao sistema de saúde o mais próximo possível da moradia, facilitando o acesso e a integração a outros equipamentos presentes no território"(32).

Com o objetivo de refletir sobre a atuação fonoaudiológica desenvolvida junto ao PSF, foi desenvolvida uma pesquisa qualitativa, baseada na análise retrospectiva da prática fonoaudiológica junto ao PSF, em uma UBS de São Paulo, com diferentes tipos de atuações: terapia individual, terapia em grupo, visita domiciliar, reuniões em equipe de saúde, ações junto aos equipamentos existentes no território (creches e escolas), como também a participação junto às campanhas públicas (aleitamento materno, vacinação do idoso) com ações relacionadas à prática fonoaudiológica. Os resultados indicam que houve ampliação das práticas fonoaudiológicas, suprindo a demanda de abrangência da UBS, o que favoreceu a ampliação do acesso da população à Fonoaudiologia. As ações realizadas evidenciaram a importância das práticas fonoaudiológicas serem organizadas com base nas diretrizes do PSF, dentro de uma visão de saúde que busca a integralidade da assistência.
No entanto, observou-se que a eficiência desta integralidade depende da relação que os outros (usuários e profissionais de saúde) estabelecem com o sistema e suas diretrizes. Concluiuse que a atuação fonoaudiológica baseada nas diretrizes do PSF e a relação estabelecida entre o fonoaudiólogo, os usuários e os demais profissionais de saúde com a rede de serviços da UBS, proporcionam o acolhimento parcial das necessidades da comunidade por parte do sistema e a disseminação do acesso da população aos serviços de Fonoaudiologia ${ }^{(33)}$. Resultados muito semelhantes foram obtidos em outros estudos ${ }^{(34)}$.

Cada equipe de NASF tem uma reunião semanal para organizar e planejar o trabalho, que inclui discussões de casos e definição de projetos. As demandas e necessidades das equipes de saúde, sejam trazidas ou identificadas como prioritárias, apontam o caminho. Divididos em duplas de referência, os profissionais fazem a interlocução do trabalho de cada equipe de saúde da família com os demais integrantes da equipe de apoio. O objetivo, como um todo, é elaborar um "projeto terapêutico singular que vise uma assistência mais ampliada, pautada em produção de cuidado e melhoria de qualidade de vida e não focada na clínica de especialidade. O enfoque principal é a promoção da saúde, e não apenas a cura de doenças. As ações dos núcleos são vinculadas àquelas já desenvolvidas pelas equipes de saúde da família ${ }^{(21)}$.

A implantação dos NASFs vêm levantando várias questões. Uma reflexão que surge, a partir deste momento, refere-se à formação dos profissionais que atuam nos núcleos, ou seja, à adequação, ou não, dos currículos dos cursos à nova realidade da Saúde Pública do país. A Educação Continuada faz parte do universo de todos os núcleos. É de extrema importância que as equipes de NASF tenham encontros periódicos para compartilhar saberes, discutir temas específicos e textos relativos à prática na ESF. Mas os recentes rumos da política de saúde do país demandam adaptações já no decorrer da graduação. Os profissionais que deixam as escolas devem estar aptos a atender uma grande gama de casos e a dar prioridade a atividades multidisciplinares de projetos terapêuticos para atuar neste novo contexto. Novas formações na graduação, cursos de residência multiprofissional no âmbito do SUS/ESF/NASF e novos cursos de pós-graduação nesta área também são muito importantes $^{(21)}$.

O documento também alerta para a necessidade do compromisso com a inclusão social, o que leva ao fortalecimento dos serviços de atenção primária para o cuidado da população com deficiência. E determina que "suas equipes tenham os conhecimentos necessários à realização de uma atenção resolutiva e de qualidade, encaminhando adequadamente os usuários para os outros níveis de complexidade, quando se fizer necessário ${ }^{(25)}$.

Ainda segundo a portaria, "as ações de reabilitação devem ser multiprofissionais e transdisciplinares, provendo o desenvolvimento de responsabilidades compartilhadas no qual, por meio do entrosamento constante entre os diferentes profissionais, se formulem projetos terapêuticos únicos que considerem a pessoa, suas necessidades e o significado da deficiência no contexto familiar e social". Os resultados das ações deverão ser constantemente avaliados na busca por ações mais adequadas e prover o melhor cuidado longitudinal aos usuários"(32). 
Como a demanda no setor de Fonoaudiologia é muito grande e na maioria das vezes, é maior que a disponibilidade de recursos humanos, tecnológicos ou financeiros, entre outros, torna-se imprescindível priorizar necessidades ${ }^{(35)}$.

A Fonoaudiologia na Saúde Pública tem apresentado crescimento e vários estudos já demonstraram a elevada ocorrência de distúrbios da comunicação em pessoas que recorrem aos serviços públicos. Acredita-se que chegou o momento de se fazer um levantamento do que está sendo feito em termos de prevenção e tratamento dos distúrbios da comunicação. É preciso, pois, tomar providências para suprir a alta demanda e adequar os serviços à realidade atual. Isto requer maior número de fonoaudiólogos atuando na Saúde Pública, profissionais preparados para lidar com Saúde Pública e mais atenção do Governo ao que se refere às políticas públicas em Fonoaudiologia.Atualmente, a atenção dispensada à Fonoaudiologia voltada para uma visão preventiva e coletiva ainda é deficiente. Precisa-se investir em pesquisas nessa área a fim de fundamentar a importância destas ações ${ }^{(21,36)}$.

\section{DISCUSSÃO}

Para todas as profissões o desafio principal consiste em criar a possibilidade da atuação conjunta, integrada e intersetorial, que incorpore a participação dos usuários e traduza a nova concepção ampliada de saúde assumida pelo SUS. Ocorre que esse trabalho em equipe, para a maior parte dos profissionais, não é focado na graduação e deverá ser aprendido no cotidiano da produção das ações de saúde e na imersão no trabalho coletivo e no território onde a vida acontece. Permeabilidade, inovação e plasticidade são alguns dos pré-requisitos para romper com a visão hierarquizada, corporativa e parcializada do trabalho em equipe, necessária e imprescindível para realizar esse projeto. Assim, podemos concluir que a responsabilidade sobre o sucesso dos NASF será de todos os atores envolvidos em sua construção.

O NASF desenhou minimamente uma organização do trabalho em cada local, acolheu demandas mais angustiantes das equipes, desenvolveu diagnóstico e planejamento nos territórios em que foi possível, mas para o futuro, o trabalho tende a avançar em estratégias com maior delineamento do trabalho por linhas de cuidado e redes de cuidado. Os próximos desafios estão relacionados com a estrutura da rede de saúde nos níveis secundário e terciário e com a estrutura da rede intersetorial.

É natural que tudo o que é novo provoque muitas dúvidas e incertezas, crises, adaptações e aprendizagens. Problemas surgem, e o principal deles, no meu ponto de vista, é uma quebra do paradigma do atendimento individual para as propostas de ações coletivas. Neste caso, os problemas podem estar nos próprios profissionais que estão compondo as equipes dos NASF, os quais tem dificuldades para realizar trabalhos interdisciplinares e de ações coletivas, mas também nas próprias Unidades Básicas de Saúde das regiões onde as equipes de NASF vão atuar, pois os profissionais que já atuam nas mesmas muitas vezes esperam mais um profissional para atendimento individual, o que sai fora do contexto coletivo. Outro problema tão grave quanto é quando algumas coordenações não entendem e não lideram as equipes de NASF para um trabalho interdisciplinar de promoção da saúde, o que certamente pode levá-las para o abismo. As equipes de NASF não chegaram para suprir necessidades individuais de falta de profissionais nas Unidades Básicas de Saúde e os profissionais que compõe estas equipes devem ter embasamento para trabalhos coletivos e, muitas vezes, mesmo na ausência de conhecimento para trabalhos coletivos, deve-se ter desejo de aprender e coragem em mudar suas atuações. Sem contar nos coordenadores, que devem ser os grandes líderes deste processo de mudança e atuação. Passada esta fase de formação de uma boa equipe, os problemas referentes às ações e procedimentos deste tipo de trabalho interdisciplinar são fatos presentes. Questões como padrões de cobrança de produtividade que não servem diretamente para os NASF, a insistência na ênfase em trabalhos individuais em detrimento aos trabalhos coletivos, a falta de estratégias para atuação no âmbito coletivo e de sistemas de avaliação adequados para mensurar resultados, podem nos levar para um futuro incerto.

\section{COMENTÁRIOS FINAIS}

É de extrema relevância a edição da Portaria Ministerial do NASF que institui o papel do Fonoaudiólogo, entre outros profissionais de saúde, na Atenção Básica. Além de incentivo e aporte financeiro aos municípios que implementarem tais serviços, devemos assistir a uma importante ampliação de acesso da população aos cuidados de saúde mental, reabilitação dos distúrbios da comunicação e das deficiências físicas, auditivas, mentais e visuais; orientações alimentares e de nutrição; cuidados com o corpo e acompanhamento das atividades físicas.

Abre-se, portanto, uma excelente oportunidade de contribuir de modo efetivo com a melhoria das condições de vida da população, através dos cuidados de sujeitos e grupos sociais em situação de risco e vulnerabilidade social, que antes não tinham acesso a este profissional, nem tais demandas estavam equacionadas de modo minimamente satisfatório no sistema de saúde brasileiro.

A análise das experiências de atuação da Fonoaudiologia na Atenção Básica, na Saúde da Família e, mais recentemente, nos NASF merece ser acompanhada e avaliada de perto para que possamos avançar na produção e sistematização de práticas de cuidado a saúde que respondam às necessidades da população e que sejam capazes de enfrentar os graves efeitos de distúrbios de linguagem, voz, motricidade oral e audição.

Faz-se necessário monitoramento e sistematização dos limites e potencialidades deste modelo, visando seu aprimoramento e apropriação do mesmo como estratégia de atenção integral, de inserção da Fonoaudiologia no SUS, colocando em cena a criação de redes de cuidado intersetoriais. 


\begin{abstract}
In order to provide subsidies to the analysis of the speech-language and hearing pathologist work in Primary Care, specifically in the Nucleus of Support for Family Health (NASF), this study conducted a literature review on the subject using the search tools of Medline, SciELO and LILACS, through the simultaneous advanced database search of the Virtual Health Library - Bireme. The study briefly recovered the history of Speech-Language and Hearing Pathology on the Brazilian's national health system (Unified Health System - SUS), and emphasized the importance of the Family Health Strategy related to Speech-Language and Hearing Pathology services in the NASFs. The literature review indicates that the performance of Speech-Language and Hearing Pathology within the health system is quite extensive, spanning different levels of assistance, which requires the development of different technologies of health work. Actions developed by NASFs necessarily require the performance of interdisciplinary teams, involved in interdisciplinary work processes and in the creation/invention of therapeutic strategies. The analysis of Speech-Language and Hearing Pathology's experiences in Primary Health Care, in Family Health Care and, more recently, in NASFs, deserves to be closely monitored and evaluated, so the production and systematization of health care practices can advance to attend the population needs, and can be able to face the serious effects of language, voice, oral motor and hearing disorders. Thus, it is necessary to monitor and to systematize the limits and potentials of this model, aiming its improvement and ownership as a strategy for the integration of Speech-Language and Hearing Pathology in the SUS.
\end{abstract}

Keywords: Public health; Primary health care; Family healh; Single health system; Health promotion

\section{REFERÊNCIAS}

1. Guarino HA, Mendes VLF. Análise da eficácia do trabalho fonoaudiológico junto ao programa de saúde da família: um estudo de caso. In: $16^{\circ}$ Congresso Brasileiro de Fonoaudiologia; 2008; set 24-27; Campos do Jordão. Rev Soc Bras Fonoaudiol. 2008;(Supl Esp):1047.

2. Brasil. Ministério da Saúde. Secretária de Atenção à Saúde. Departamento de Atenção Básica. Política Nacional de Atenção Básica. Brasília: Ministério da Saúde; 2006.

3. Van Stralen CJ, Belisário SA, Van Stralen TBS, Lima AMD, Massote AW, Oliveira CL. Percepção dos usuários e profissionais de saúde sobre atenção básica: comparação entre unidades com e sem saúde da família na Região Centro-Oeste do Brasil. Cad Saúde Pública = Rep Public Health. 2008; 24(Supl 1):S148-58.

4. Bonamigo AW, Rodrigues PVP. Aspectos históricos e conceituais do programa saúde da família ao NASF. In: $17^{\circ}$ Congresso Brasileiro de Fonoaudiologia; 2009; out 21-24; Salvador. Rev Soc Bras Fonoaudiol. 2009;(Supl Esp):2382.

5. Mendes VLF. Fonoaudiologia, Atenção Básica e Saúde da Família. In: Fernandes FDM, Mendes BCA, Navas AL. Tratado de fonoaudiologia. 2a ed. São Paulo: Roca; 2009. p. 612-8.

6. Mendes VLF. Atuação fonoaudiológica no NASF. In: $17^{\circ}$ Congresso Brasileiro de Fonoaudiologia; 2009; out 21-24; Salvador. Rev Soc Bras Fonoaudiol. 2009; (Supl Esp).

7. Kruk ME, Porignon D, Rockers PC, Lerberghe WV. The contribution of primary care to health and health systems in low- and middle-income countries: a critical review of major primary care initiatives. Soc Sci Med. 2010;70(6):904-11.

8. Mendonça CS. Saúde da Família, agora mais do que nunca! Ciênc Saúde Coletiva. 2009;14(Supl 1):1493-7.

9. Macinko J, de Souza Mde F, Guanais FC, da Silva Simões CC. Going to scale with community-based primary care: an analysis of the family health program and infant mortality in Brazil, 1999-2004. Soc Sci Med. 2007;65(10):2070-80.

10. NASF: Fonoaudiologia conquista espaço na saúde pública. J CFFa. 2008;9(36):4-5.

11. Forster AC, Rocha JSY, Almeida TL, Guedes RB, Dal Fabbro AL, Caccia-Bava MCGG. Prácticas de gestión en la atención básica y en el programa de salud de la familia. Aten Primaria. 2008;40(5):265-6.

12. Ronzani TM, Silva CM. O Programa Saúde da Família segundo profissionais de saúde, gestores e usuários. Ciênc Saúde Coletiva. 2008;13(1):23-34

13. Resta DG, Motta MGC. Família em situação de risco e sua inserção no Programa de Saúde da Família: uma reflexão necessária à pratica profissional. Texto \& Contexto Enferm. 2005;14(N Esp):109-15.

14. Centro de Estudos em Cultura Contemporânea, Consórcio Medicina USP. Inquérito com usuários e profissionais, percepção dos gestores e estudos sobre o trabalho no PSF. São Paulo: CEDEC: Consórcio Medicina USP; 2006. p. 129-72.

15. Costa MFBNA, Ciosak SI, Bartolomé AG, Isern MTI. La integralidad en la atención primaria de salud en los sistemas de salud brasileño y español. Aten Primaria. 2009;41(7):402-4.

16. Pujol Ribera E, Badia JG, Corralesa MS, Sampietro-Colome L, Rua MIP, Iglesias-Pérez B, et al. El producto de la atención primaria definido por profesionales y usuarios. Gac Sanit. 2006;20(3):209-19.

17. Araújo MBS, Rocha PM. Saúde da família: mudando práticas? Estudo de caso no município de Natal (RN). Ciênc Saúde Coletiva. 2009;14(Supl 1):1439-52.

18. Bertoldi AD, de Barros AJ, Wagner A, Ross-Degnan D, Hallal PC. Medicine access and utilization in a population covered by primary health care in Brazil. Health Policy. 2009;89(3):295-302.

19. Cavalheiro MTP. Fonoaudiologia e saúde da família. Rev CEFAC. 2009;11(2):4-5.

20. Mângia EF, Lancman S. Núcleos de apoio à saúde da família: integralidade e trabalho em equipe multiprofissional. Rev Ter Ocup. 2008;19(2):i-i.

21. Florindo AA. Núcleos de Apoio à Saúde da Família e a promoção das atividades físicas no Brasil: de onde viemos, onde estamos e para onde vamos. Rev Bras Ativ Fís Saúde. 2009;14(2):5-6.

22. Mendes VLF, Cardoso F, Magalhães LA, Zorzi DS, Campos MF, Guedes SDR, Souza LAP. Ações de matriciamento no NASF: propostas de intervenção na atenção básica. In: $17^{\circ}$ Congresso Brasileiro de Fonoaudiologia; 2009; out 21-24; Salvador. Rev Soc Bras Fonoaudiol. 2009;(Supl Esp):1574.

23. Santos RBP, Mancopes R, Terçariol D, Urnau D, Girardi D, Oliveira IC. Participação da Fonoaudiologia no Núcleo de Apoio à Saúde da Família: relato de experiência na UNIVALI -SC. In: $17^{\circ}$ Congresso Brasileiro de Fonoaudiologia; 2009; out 21-24; Salvador. Rev Soc Bras Fonoaudiol. 2009;(Supl Esp):1778.

24. Klock AD, Heck RM, Casarim ST. Cuidado domiciliar: a experiência da residência multiprofissional em saúde da família/UFPEL-MS/BID. Texto e Contexto Enferm. 2005;14(2):237-45.

25. NASF: previsão de 500 vagas para fonoaudiólogos até 2011. J CFFa. 2008;9(39):4-5. 
26. Mendes VLF. Uma clínica no coletivo: experimentações no Programa de Saúde da Família. São Paulo: Hucitec; 2007. 165p. (Saúde Loucura, 21)

27. Rezende M, Moreira MR, Amâncio Filho A, Tavares MFL. A equipe multiprofissional da 'Saúde da Família': uma reflexão sobre o papel do fisioterapeuta. Ciênc \& Saúde Coletiva. 2009;14(Supl 1):1403-10.

28. Gurgueira AL. Fonoaudiologia no Sistema Único de Saúde. In: Fernandes FDM, Mendes BCA, Navas AL. Tratado de fonoaudiologia. 2a ed. São Paulo: Roca; 2009. p. 619-26.

29. Ferreira JM, Pimentel ARS, Silva CAB, Silva FB. Núcleo de Apoio à Saúde da Família: relato de experiência da fonoaudiologia em políticas públicas. In: $17^{\circ}$ Congresso Brasileiro de Fonoaudiologia; 2009; out 21-24; Salvador. Rev Soc Bras Fonoaudiol. 2009;(Supl Esp):2405.

30. Buccini GS, Sanches MTC, Nogueira-Martins MCF, Bonamigo AW. Conhecimentos e práticas das equipes de saúde de família sobre o aleitamento materno no seguimento ambulatorial dos RNBP pertencentes à região de Pirituba Perus - São Paulo. In: $16^{\circ}$ Congresso Brasileiro de Fonoaudiologia; 2008; set 24-27; Campos do Jordão. Rev Soc Bras Fonoaudiol. 2008;(Supl Esp):1272.
31. Namen VC, Garcia GM. Demanda de casos específicos para o fonoaudiólogo do núcleo de apoio à saúde da família (NASF) na região sudeste do município de São Paulo. In: $17^{\circ}$ Congresso Brasileiro de Fonoaudiologia; 2009; out 21-24; Salvador. Rev Soc Bras Fonoaudiol. 2009;(Supl Esp):1936.

32. Brasil. Ministério da Saúde. Portaria $\mathrm{n}^{\circ} 154$ de 24 de janeiro de 2008. Cria os Núcleos de Apoio à Saúde da Família - NASF. Brasília: Ministério da Saúde; 2008.

33. Araújo MLB, Freire RMAC. Relato de uma experiência da atuação fonoaudiológica no programa de saúde da família (PSF). In: $16^{\circ}$ Congresso Brasileiro de Fonoaudiologia; 2008; set 24-27; Campos do Jordão. Rev Soc Bras Fonoaudiol. 2008;(Supl Esp):1096.

34. Carvalho RS. Atuação fonoaudiológica no NASF - Núcleo de Apoio à Saúde da Família. In: $17^{\circ}$ Congresso Brasileiro de Fonoaudiologia; 2009; out 21-24; Salvador. Rev Soc Bras Fonoaudiol. 2009;(Supl Esp): 1807 .

35. Goulart BNG, Chiari BM. Construção e aplicação de indicadores de saúde na perspectiva fonoaudiológica: contribuições para reflexão. Rev Soc Bras Fonoaudiol. 2006;11(3):194-204.

36. Moreira MD, Mota HB. Os caminhos da fonoaudiologia no Sistema Único de Saúde - SUS. Rev CEFAC. 2009;11(3):516-21. 